\title{
(C) OPEN ACCESS \\ Speaking up about care concerns in the ICU: patient and family experiences, attitudes and perceived barriers
}

\author{
Sigall K Bell, ${ }^{1}$ Stephanie D Roche, ${ }^{2}$ Ariel Mueller, ${ }^{3}$ Erica Dente, ${ }^{4}$ \\ Kristin O'Reilly, ${ }^{2,5}$ Barbara Sarnoff Lee, ${ }^{6}$ Kenneth Sands, ${ }^{1,2,5}$ \\ Daniel Talmor, ${ }^{3,5}$ Samuel M Brown ${ }^{7,8}$
}

- Additional material is published online only. To view please visit the journal online (http://dx.doi.org/10.1136/ bmjas-2017-007525).

For numbered affiliations see end of article.

\section{Correspondence to}

Dr Sigall K Bell, Department of Medicine, Beth Israel Deaconess Medical Center, Boston, MA 02215, USA;

sbell1@bidmc.harvard.edu

SKB and SDR contributed equally.

Received 23 October 2017 Revised 23 May 2018 Accepted 27 May 2018 Published Online First 12 July 2018

\section{Check for updates}

To cite: Bell SK, Roche SD Mueller A, et al. BMJ Qual Saf 2018;27:928-936.

\begin{abstract}
Background Little is known about patient/family comfort voicing care concerns in real time, especially in the intensive care unit (ICU) where stakes are high and time is compressed. Experts advocate patient and family engagement in safety, which will require that patients/families be able to voice concerns. Data on patient/family attitudes and experiences regarding speaking up are sparse, and mostly include reporting events retrospectively, rather than pre-emptively, to try to prevent harm. We aimed to (1) assess patient/family comfort speaking up about common ICU concerns; (2) identify patient/family-perceived barriers to speaking up; and (3) explore factors associated with patient/family comfort speaking up.
\end{abstract}

Methods In collaboration with patients/families, we developed a survey to evaluate speaking up attitudes and behaviours. We surveyed current ICU families in person at an urban US academic medical centre, supplemented with a larger national internet sample of individuals with prior ICU experience.

Results 105/125 (84\%) of current families and 1050 internet panel participants with ICU history completed the surveys. Among the current ICU families, $50 \%-70 \%$ expressed hesitancy to voice concerns about possible mistakes, mismatched care goals, confusing/ conflicting information and inadequate hand hygiene. Results among prior ICU participants were similar. Half of all respondents reported at least one barrier to voicing concerns, most commonly not wanting to be a 'troublemaker', 'team is too busy' or 'I don't know how'. Older, female participants and those with personal or family employment in healthcare were more likely to report comfort speaking up.

Conclusion Speaking up may be challenging for ICU patients/families. Patient/family education about how to speak up and assurance that raising concerns will not create 'trouble' may help promote open discussions about care concerns and possible errors in the ICU.

\section{INTRODUCTION}

Although patients and families are increasingly considered part of the 'care team' and encouraged to participate in care and in safety, ${ }^{1-3}$ little is known about patient and family comfort with voicing concerns in real time to clinicians. Yet fostering comfort for patients and families to voice concerns is central to patient-centred care ${ }^{4}$ including understanding the risks and benefits of care and participating in shared decision-making and informed consent. In addition, speaking up about care concerns may contribute to patient safety as part of an overall safety culture. ${ }^{5}$ For example, families-vigilant stakeholders-may be the first to detect a change in a patient's clinical status. Also, as the 'common thread' uniting care encounters, patients and families may improve continuity of care among team members, or convey key information that clinicians may have overlooked. ${ }^{6}$ Finally, patients and families can detect errors, even those not apparent to clinicians. ${ }^{7-10}$

Supporting patients and families to voice concerns may be particularly important in the intensive care unit (ICU) ${ }^{11}$ which provides high-intensity, high-acuity, errorprone and preference-sensitive care to millions of patients annually, amounting to $13 \%$ of hospital costs and $4 \%$ of national health expenditures in the USA. ${ }^{12}$ But voicing concerns in the ICU can be problematic for patients and families: the physical environment may be perceived as strange or dangerous, patients are at high risk of death, power asymmetries are extreme, multiple clinicians and teams are involved, and the complexity of care may be overwhelming. Patients and families may hesitate to confront clinicians-the individuals they rely on for treatmentand they may fear negative repercussions if they speak up. ${ }^{13}{ }^{14}$ Acute illness may also decrease comfort voicing concerns. ${ }^{15} 16$ 
At the same time, staffing ratios may enable closer relationships between families and clinicians, with unpredictable effects on speaking up. Holding one's voice may have traumatic consequences for patients and families, including guilt and loss stemming from preventable errors or deaths, suffering unwanted care, poor quality of dying, or psychological injury as a part of post-ICU syndrome. ${ }^{17-21}$

The potentially devastating effects of 'silence' when speaking up is needed ${ }^{22}$ have led to focused attention on speaking up as part of safety culture. ${ }^{24} 25$ Although speaking up among clinicians improves team performance, ${ }^{26} 27$ outcomes have been less thoroughly studied for patients and families. ${ }^{28-32}$ Nonetheless, empowering patients and families to 'stop the line' when something is wrong seems prudent, particularly given their unique knowledge of the patient, accounts of families who 'knew something was not right' before a harmful event, ${ }^{14}{ }^{33}$ and the potential to prevent catastrophic outcomes such as serious medication errors. ${ }^{34}$ Safety and engagement experts identify assessment of patient and family comfort with speaking up as a critical next step in improving safety culture, ${ }^{45}$ building on recent metrics developed for clinicians ${ }^{25} 36$ and initiatives that encourage patients and families to raise concerns about possible care breakdowns and mistakes. ${ }^{37-39}$ While conceptual models of voice and silence have proven multidimensional and complex, ${ }^{25} 4041$ factors influencing voice among professionals have been described. ${ }^{42}{ }^{43}$ These include hierarchy, lack of experience or skills speaking up, quality of relationships among involved persons, clinical environment characteristics, psychological safety, and personal factors such as assertiveness. ${ }^{43-49}$ Although several of these factors may apply to patients and families, other unique issues may play a role, and this question is not well studied. We therefore aimed to assess patient/family comfort voicing concerns in a high-stakes, dynamic environment. We approached the problem in a collaborative manner that included members of our patient and family advisory council (PFAC) as partners from beginning to end, working to address three core research questions:

1. What proportion of ICU patients/families report feeling comfortable voicing concerns about common ICU issues?

2. What are the most common patient and family self-reported barriers to voicing concerns in the ICU?

3. What patient/family factors are associated with greater likelihood of self-reported comfort voicing concerns in the ICU?

\section{METHODS}

\section{Participants}

\section{Current ICU family cohort}

From July 2014 to February 2015, we conducted a survey of adult family members of patients admitted to the ICU at our north-east US urban academic medical centre. The survey was administered by trained research assistants who visited the ICUs and ICU waiting rooms hourly between 09:00 and 18:00 on weekdays to identify available family members who met the eligibility criteria: adults (ages 18-89) living in the USA who could communicate in English and had a loved one admitted to the ICU. Family members who agreed to participate were given an electronic tablet on which to complete the survey independently, and the research assistant returned to collect the tablet when the participant finished.

\section{ICU admission history—internet panel participants}

Because our current ICU family cohort was limited to a single hospital in the north-east USA, we identified a national internet cohort of individuals with self-reported ICU experience within the last 10 years, to whom we administered the same survey. Given expected differences related to mode of administration, acuity of exposure to the ICU experience and broader cultural differences, we did not compare the two cohorts statistically: the internet cohort was intended to qualitatively confirm and extend our findings from the current ICU family cohort, with a more feasible approach to enrolment of a large number of respondents. The internet-based survey was administered via Qualtrics (Provo, Utah), an internet survey firm that uses standard empanelment procedures to recruit sample populations for marketing, quality assurance and medical research. ${ }^{50}$

\section{Survey design}

We developed the survey instrument collaboratively with representatives from the medical centre's PFAC and a multidisciplinary team of experts in communication, patient engagement, ICU care, clinical research and patient safety. In this paper, we report the results of survey items focused on patient and family comfort voicing concerns in the ICU setting. Our detailed, iterative survey development methodology, along with the results of other items and/or instruments administered during the overall survey, has been published elsewhere. ${ }^{51}$ We conducted cognitive testing of survey items with PFAC members and iteratively improved the survey via seven subsequent reviews by the PFAC and content experts. Several attention questions were built into the final survey, such that responses with incorrect answers were excluded. Surveys with more than 20\% missing items were also excluded.

With input from our PFAC members, we developed survey items evaluating patient and family comfort voicing concerns about eight common ICU topics, such as medications, conflicting or confusing information, and concern about a possible mistake. We asked respondents the extent to which they would feel comfortable speaking up in these situations using a 4-point Likert-style item ranging from 'very uncomfortable' to 'very comfortable'. We also asked why participants might hesitate to voice concerns, using 
items identified from a literature review and discussions with patient/family advisors. ${ }^{5}{ }^{17-22} 52$ While some clinical scenarios call for voicing concerns as part of patient-centred care, others, such as notifying clinicians about possible mistakes, involve voicing concerns as part of safe care. We use the term 'speaking up' for simplicity when referring to the collective comfort with voicing concerns to the care team about several aspects of ICU care, some of which include safety issues. We collected demographic information including age, sex, ethnicity, education, and whether the participant, or a close friend or family member, works in healthcare. Relevant portions of the survey instrument are available in the online supplementary appendix A.

\section{Statistical methods}

Using SAS V.9.3, we calculated the descriptive statistics of the data. For the first research question-patient and family comfort voicing concerns in eight ICU conversations-we conducted univariate analysis of the eight speaking up items. For this analysis, we dichotomised 'very comfortable' versus remaining responses since all other answer choices indicated some degree of hesitancy to voice concerns. We analysed responses to each item separately to assess any variance in patient-reported and family-reported comfort voicing concerns about each of these ICU conversations.

For the second research question-barriers to voicing concerns-we used descriptive statistics to summarise the number of and the most common barriers selected by participants.

For the third research question-patient and family factors associated with comfort voicing concerns-we first assessed the possibility of summarising the eight speaking up items (measured on a 4-point Likert-style scale) as a single 'Speaking Up' score through principal component analysis (PCA) and calculation of Cronbach's alpha to test internal consistency. On PCA of the speaking up items, only the first principal component was retained, and it accounted for $55 \%$ of the variance (see online supplementary appendix B). Cronbach's alpha was 0.88 , suggesting high internal consistency. Based on these results, we collapsed the eight items into a single summary score and used this measure as the outcome of interest for multiple linear regression. We used the adjusted $\mathrm{R}^{2}$ method for model selection targeted to Akaike information criteria. ${ }^{53}$ Our prespecified candidate variables (based on available demographic data) included age, sex, ethnicity, education, whether the participant works in healthcare, or had close friends or family that work in healthcare, and the EuroQol-5 dimensions (EQ-5D) utility (a brief measure of quality of life). ${ }^{54}$

\section{Ethical considerations}

All participants provided implied consent prior to answering the survey. In-person survey participants did not receive compensation. Internet survey respondents were compensated following the standard Qualtrics method (\$1 per completed survey).

\section{RESULTS}

\section{Respondents}

A total of 105/125 (84\%) patients and family members completed the in-person surveys. In the internet panel, 2100 returned usable surveys. Among these, 1050 reported a prior ICU admission and were included in our analysis (figure 1). Respondent characteristics are displayed in table 1 . Participants were predominantly middle-aged (median age 49), female (69\%) and of white race $(85 \%)$.

\section{Comfort voicing concerns}

Among current ICU families, participants reported varying degrees of comfort voicing concerns in common ICU situations (table 2), with 69\% of families reporting they were very comfortable discussing medications and $31 \%$ reporting they were very comfortable discussing hand hygiene. About a third of current ICU family members were very comfortable discussing mismatched care goals, including disagreements where they or their loved one wanted care that was more $(31 \%)$ or less aggressive (35\%) than the team proposed. Approximately half of the current ICU family members felt very comfortable asking for clarification of confusing or conflicting information (52\%), or discussing a possible mistake in care (46\%). Findings overall showed similar trends between current families and participants reporting a prior ICU experience (table 2).

\section{Barriers to voicing concerns}

In total, 16/103 (16\%) current ICU families and $181 / 1050$ (17\%) prior ICU participants reported they did not have concerns. Among all remaining respondents, about half $(504 / 956,53 \%)$ provided at least one reason for hesitating to speak up. Invited to select up to three reasons, current ICU families selected a mean of 1.6 reasons (SD 0.81) and prior ICU participants selected a mean of 1.8 reasons (SD 0.83). Participants across both groups most commonly selected 'I don't want to be labeled as a "troublemaker" (34\%); 'the team is too busy' (32\%); 'I don't know how to raise my concern or who to talk to' (32\%); 'I am afraid of seeming like I don't understand medical concepts' (23\%); and 'I don't want to harm my relationship with members of the medical team' (21\%) (table 3). While the top 5 responses were the same across groups, compared with internet participants, family members of current ICU families more commonly reported the care team's time constraints and fear of inadequate medical knowledge as a barrier. Internet participants more commonly reported they would hesitate because they did not know how to speak up, because 'nothing would be done about my concerns' or because 'it isn't my place to question the team'. 


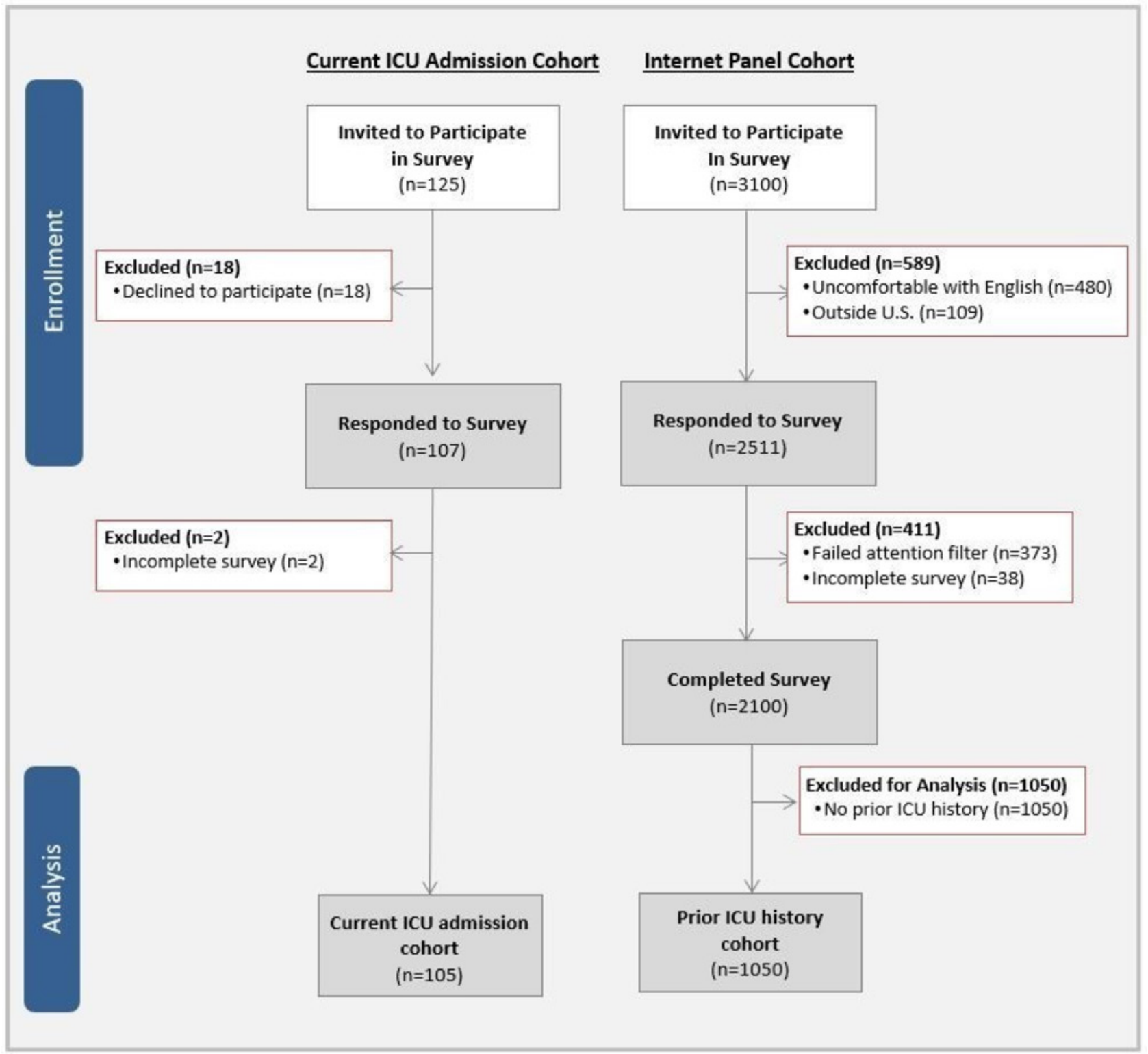

Figure 1 Participant recruitment adapted from Brown et al. ${ }^{51} \mathrm{ICU}$, intensive care unit.

\section{Patient/family factors associated with comfort voicing concerns}

In multivariable regression of the speaking up score, older age, female sex and working in healthcare (all $\mathrm{p}<0.05$ ) were positively associated with greater reported comfort voicing concerns, although with modest effects (online supplementary appendix C).

\section{DISCUSSION}

Our study of patient and family comfort speaking up in the ICU-the first to our knowledge to address this issue-revealed that challenges to speaking up may be substantial during an ICU stay. Overall, $50 \%-70 \%$ of current ICU admission patients and families expressed hesitancy to voice concerns in real time about some common care situations with safety implications, including a possible mistake or mismatched goals related to aggressiveness of care desired by patient/family versus delivered by the care team. We were surprised to find substantial deficits in patient/family comfort voicing concerns, even in 'life or death' settings of the ICU, where the need to speak up may be particularly dire. Patients and family reported some barriers to comfort speaking up in the ICU that were similar to clinicians' and some that were unique. ${ }^{24} 434755$ Although the study focused on families at one north-east US hospital, overall responses were similar among respondents in a national internet panel of patients and families with prior ICU admissions across the USA. Given the high risks of errors, the exquisite preference sensitivity and the fraught dynamics of the ICU, we estimate that the ICU is one of the most important settings for optimising speaking up opportunities for patients and families. 
Table 1 Participant demographics

\begin{tabular}{|c|c|c|c|}
\hline & $\begin{array}{l}\text { Overall } \\
\mathrm{n}=1155\end{array}$ & $\begin{array}{l}\text { Current ICU admission } \\
\mathrm{n}=105\end{array}$ & $\begin{array}{l}\text { Prior ICU admission } \\
\mathrm{n}=1050\end{array}$ \\
\hline Age (years)* & $47.3(14.1)$ & $51(15.9)$ & $47(13.9)$ \\
\hline Female & $790(68.4)$ & $75(71.4)$ & $715(68.0)$ \\
\hline \multicolumn{4}{|l|}{ Ethnicity } \\
\hline White/Caucasian & $979(84.8)$ & $81(77.1)$ & $898(85.5)$ \\
\hline Black/African-American & $84(7.3)$ & $8(7.6)$ & $76(7.2)$ \\
\hline Latino/Hispanic & $38(3.3)$ & $6(5.7)$ & $32(3.0)$ \\
\hline Asian & $38(3.3)$ & $7(6.7)$ & $31(3.0)$ \\
\hline Other & $12(1.0)$ & $1(1.0)$ & $11(1.0)$ \\
\hline \multicolumn{4}{|l|}{ Highest level of education } \\
\hline High school graduate (or equivalent) or less & $295(25.5)$ & $16(15.2)$ & $279(26.6)$ \\
\hline Some college (1-4 years, no degree) or associate's degree & $448(38.8)$ & $35(33.3)$ & $413(39.3)$ \\
\hline Bachelor's degree or higher & $409(35.4)$ & $52(49.5)$ & $357(34.0)$ \\
\hline Works in healthcare & $88(7.6)$ & $19(18.1)$ & $69(6.6)$ \\
\hline Close family member or friend works in healthcare & $412(35.7)$ & $72(68.6)$ & $340(32.4)$ \\
\hline EuroQol-5 dimensions (EQ-5D) utility index* & $0.8(0.2)$ & $0.9(0.2)$ & $0.8(0.2)$ \\
\hline EuroQol-5 dimensions (EQ-5D) Visual Analogue Scale* & $74.1(19.9)$ & $84(18.4)$ & $73(19.8)$ \\
\hline \multicolumn{4}{|l|}{ Region } \\
\hline North-east & $315(27.3)$ & $105(100)$ & $210(20.0)$ \\
\hline Midwest & - & - & $289(27.5)$ \\
\hline South & - & - & $355(33.8)$ \\
\hline West & - & - & $189(18.0)$ \\
\hline Unknown & - & - & $7(0.7)$ \\
\hline
\end{tabular}

*Presented as mean (SD); the remaining variables are presented as $\mathrm{n}(\%)$, using the denominator indicated in the column header. $\mathrm{ICU}$, intensive care unit.

Using a survey instrument developed with patients and families to reflect what matters most to them in terms of challenging ICU conversations and barriers to voicing concerns, our data provide key new evidence for healthcare organisations and clinicians to consider as they design and evaluate interventions to improve safety, quality and patient-centred care in the ICU, and have several practice implications. First, true partnerships with patients and families may be limited if they do not feel comfortable voicing concerns. Initiatives

Table 2 Proportion of participants reporting they were 'very comfortable' speaking up in specific ICU conversations

\begin{tabular}{|c|c|c|c|}
\hline ICU conversation* & $\begin{array}{l}\text { Overall } \\
\mathrm{n}=1155\end{array}$ & $\begin{array}{l}\text { Current } \\
\text { ICU admission } \\
n=105\end{array}$ & $\begin{array}{l}\text { Prior } \\
\text { ICU admission } \\
n=1050\end{array}$ \\
\hline \multicolumn{4}{|l|}{ How comfortable would you be asking a member of your medical team: (\% very comfortable) } \\
\hline $\begin{array}{l}\text { To review with you the name, dose or reason for medications when they are administered (to be } \\
\text { sure they are correct)? }\end{array}$ & $811(70.2)$ & $72(68.6)$ & $739(70.4)$ \\
\hline Whether he/she has cleaned his/her hands? & $481(41.6)$ & $32(30.5)$ & $449(42.8)$ \\
\hline For a meeting with the healthcare team to review your loved one's health status? & $726(62.9)$ & $58(55.2)$ & $668(63.6)$ \\
\hline About concerns you may have regarding your loved one's medical care? & $779(67.4)$ & $61(58.1)$ & $718(68.4)$ \\
\hline $\begin{array}{l}\text { To discuss a disagreement where you or your loved one wants less aggressive medical care than } \\
\text { the team is proposing? }\end{array}$ & $520(45.0)$ & $37(35.2)$ & $483(46.0)$ \\
\hline $\begin{array}{l}\text { To discuss a disagreement where you or your loved one wants more aggressive medical care than } \\
\text { the team is proposing? }\end{array}$ & $504(43.6)$ & $33(31.4)$ & $471(44.9)$ \\
\hline About a possible mistake in your loved one's medical care? & $624(54.0)$ & $48(45.7)$ & $576(54.9)$ \\
\hline To clarify if he/she gives you confusing information? & $746(64.6)$ & $55(52.4)$ & $691(65.8)$ \\
\hline \multicolumn{4}{|l|}{ Overall comfort } \\
\hline Speaking up summary scoret & $27.3(4.4)$ & $26.0(5.5)$ & $27.4(4.2)$ \\
\hline
\end{tabular}


Table 3 Reasons for hesitating to voice concerns among respondents who had concerns

\begin{tabular}{|c|c|c|c|}
\hline & $\begin{array}{l}\text { Overall } \\
\mathrm{n}=504\end{array}$ & $\begin{array}{l}\text { Current ICU } \\
\text { admission } \\
\mathrm{n}=29 \text { * }\end{array}$ & $\begin{array}{l}\text { Prior } \\
\text { ICU admission } \\
\mathrm{n}=475 t\end{array}$ \\
\hline \multicolumn{4}{|l|}{ Reason(s) I hesitate to tell a member of the medical team about concernsł } \\
\hline I don't want to be labelled as a 'troublemaker'. & $173(34.3)$ & $9(31.0)$ & $164(34.5)$ \\
\hline The team is too busy for an extra discussion. & $162(32.1)$ & $12(41.4)$ & $150(31.6)$ \\
\hline I don't know how to raise my concern or who to talk to. & $159(31.5)$ & $6(20.7)$ & $153(32.2)$ \\
\hline I am afraid of seeming like I don't understand medical concepts. & $114(22.6)$ & $9(31.0)$ & $105(22.1)$ \\
\hline I don't want to harm my relationship with members of the medical team. & $104(20.6)$ & $7(24.1)$ & $97(20.4)$ \\
\hline Nothing would be done about my concerns. & $92(18.3)$ & $1(3.4)$ & $91(19.2)$ \\
\hline It isn't my place to question the team. & $78(15.5)$ & $1(3.4)$ & $77(16.2)$ \\
\hline I don't want to get the healthcare provider in trouble. & $21(4.2)$ & $2(6.9)$ & $19(4.0)$ \\
\hline Other§ & $19(3.8)$ & $3(10.3)$ & $16(3.4)$ \\
\hline \multicolumn{4}{|c|}{$\begin{array}{l}\text { *In the current family cohort, } 2 / 105 \text { individuals did not respond to this question and 16/103 participants did not have concerns. Among the remaining } \\
\text { respondents, } 29 / 87 \text { who had concerns indicated one or more reasons why they would hesitate to speak up and } 58 / 87 \text { indicated that they would not } \\
\text { hesitate to speak up. } \\
\text { †Among internet participants, } 181 / 1050 \text { did not have concerns. Among the remaining respondents, } 475 / 869 \text { individuals who had concerns indicated one } \\
\text { or more reasons why they would hesitate to speak up and } 394 / 869 \text { indicated that they would not hesitate to speak up. } \\
\text { łPresented as percentages, using the } n \text { indicated in the column header as the denominator, representing the number of respondents who selected at } \\
\text { least one reason to hesitate. Percentages do not sum to } 100 \text { because participants could select up to three responses. } \\
\S^{\prime} \text { Other' responses included confusion or inability to remember questions, shy personality, perception that team is not interested or doesn't care, fear of } \\
\text { retribution and lack of privacy/family dynamics. } \\
\text { ICU, intensive care unit. }\end{array}$} \\
\hline
\end{tabular}

to promote shared decision-making (particularly about care goals), family involvement in interdisciplinary rounds, and other elements of patient and family engagement may have limited impact if they do not also actively and intentionally support voicing concerns in real time.

Second, our findings highlight specific clinical situations that merit focused attention. In addition to hand hygiene, patients and families were least comfortable speaking up about disagreements where they/ their loved one wanted more or less aggressive care than the team was proposing. Supporting patients and families to voice their priorities could potentially decrease patient/family distress and, in some cases, even healthcare costs associated with inappropriately aggressive care. ${ }^{56}$ The reported reluctance to speak up about hand hygiene, a priority in preventing healthcare-associated infection, in both this and prior studies suggests the need for improved strategies to encourage assertiveness about this issue or alternative approaches to improve clinician hand hygiene. ${ }^{57}$ Our study participants' hesitancy to ask about 'confusing or conflicting information' may have significant consequences given its previously reported association with the quality of death and dying. ${ }^{18}$ Finally, our results highlight the need to explicitly support patients/families to speak up in real time about perceived errors. Although experts call for partnership with patients and families to prevent medical errors, ${ }^{25} 58$ only about half of respondents indicated they would readily speak up about a potential mistake. Given current interest in transparency and patient engagement in safety, helping patients and families speak up in real time is likely an underexplored and undersupported component of effective safety partnerships. ${ }^{11} 58$

Third, patient-reported barriers to voicing concerns indicate targets for improvement. We were surprised that not knowing who to talk to about concerns-a readily actionable issue-was among the most common barriers reported by patients and families, alongside 'not wanting to be a troublemaker'. Clear and systematic instructions about whom to contact with concerns are a critical first step. Culturally reframing speaking up more positively-from 'causing trouble' to being an 'engaged team member' or 'upstander'-may also help. ${ }^{13}$ Our findings suggest that patients and families may need greater support to offset concerns about potential negative relational repercussions from speaking up, similar to findings from studies focused on clinicians-in-training who may hesitate to speak up because of a low perceived status in the medical hierarchy, or fear of harming relationships with team members. ${ }^{43-47}$ Previously unrecognised barriers unique to patient and family speaking up include the perception that the team is too busy to hear their concerns, and that patients/families may worry about appearing to not understand medical concepts in front of their care team. Taken together, these findings resonate with other studies demonstrating that staff encouragement may play a key role in encouraging patient participation. ${ }^{55}$ Assurance that the healthcare team wants to hear about concerns may be particularly important for current ICU families, who reported 'the team is too busy' as the most common barrier to speaking up. Exploring other personal, relational and contextual characteristics pertinent to patient/family speaking up, 
such as health literacy and health status, may also help inform organisational strategies. ${ }^{24} 57$

Educational and logistical mechanisms to help patients and families voice concerns should emphasise that safety issues are vital, even if clinicians appear busy. Education programmes that instruct family members on how to assist with the care of critically ill patients can underscore the unique knowledge held by families, the importance of speaking up and the specific mechanisms for doing so, as participating in care can foster a sense of belonging to the team or may enable discovery of clinically important information. ${ }^{60}$ Family-centred communication training for clinicians should raise awareness about barriers to speaking up, emphasise encouragement of patients and families to speak up, and prepare clinicians to listen meaningfully. ${ }^{61}$ Families may also benefit from evidence that speaking up results in meaningful change, a common influencer of speaking up behaviour among healthcare and other professionals. ${ }^{62}$ Asking patients to take a risk without visible, positive responses to feedback may be counterproductive. Supporting patients and families in these ways can also send a powerful message to patients and clinicians about institutional priorities related to transparency, inclusivity and safety partnership. ${ }^{13764-68}$

Based on our findings of factors affecting voicing concerns, younger individuals, men and those without personal connection to healthcare may especially need support. Other studies suggest that less educated or unemployed patients and those with poorer health may be less likely to speak up. ${ }^{53576768}$ Our analysis did not show the same findings, perhaps because our study was specific to the ICU, because we queried family members as well as patients, or because those with higher formal education were more likely to second-guess themselves or fear negative consequences of voicing concerns. Our reported associations with speaking up should be viewed as exploratory and further examined among larger studies of ICU families, especially in light of the modest performance of the regression model.

Our findings are limited by self-report and subject to social desirability bias. Our primary cohort of current ICU family members was relatively small and surveyed at a single site, limiting generalisability; however, the internet cohort represents a large national sample, and overall trends were consistent across both cohorts. Although we based our list of candidate variables for our regression model on a literature review and discussion with patient and family advisors, it is possible that other factors not collected in our survey influence speaking up. For example, the influence of previous hospitalisations, ICU characteristics such as including families in daily rounds, speaking up climate among clinicians, and broader factors should be further studied. Our research did not interrogate a formal theoretical model, although models describing speaking up among clinicians were used as starting points to guide the enquiry along with input from PFAC members about what matters most to patients and families. We acknowledge that participant self-report may not directly correlate with observed behaviours, and future studies assessing actual patient and family speaking up at the front lines of care and the related clinical outcomes are needed. Finally, our study was not designed to differentiate between patient ICU experience and family ICU experience. Further research may delineate differences in comfort speaking up between these two groups.

\section{CONCLUSIONS}

Patients and families report considerable hesitancy to voice concerns in common ICU conversations. About half of all patients and families had some reluctance to speak up about possible mistakes, a finding which may limit both safety and engagement efforts. Patient-perceived barriers to speaking up in the ICU are mechanistic (how to do it), contextual (team is too busy) and cultural (fear of being a troublemaker). Educational strategies should include both encouraging and supporting patients and families to voice concerns with clear steps and a genuine invitation, and preparing clinicians to respond meaningfully when patients speak up.

\section{Author affiliations}

'Department of Medicine, Beth Israel Deaconess Medical Center, Harvard Medical School, Boston, Massachusetts, USA

${ }^{2}$ Department of Health Care Quality, Beth Israel Deaconess Medical Center, Boston, Massachusetts, USA

${ }^{3}$ Department of Anesthesia, Critical Care and Pain Medicine, Beth Israel Deaconess Medical Center, Boston, Massachusetts, USA

${ }^{4}$ Patient and Family Advisory Council, Beth Israel Deaconess Medical Center, Boston, Massachusetts, USA

${ }^{5}$ Center for Healthcare Delivery Science, Beth Israel Deaconess Medical Center, Boston, Massachusetts, USA

${ }^{6}$ Department of Social Work, Beth Israel Deaconess Medical Center, Boston, Massachusetts, USA

${ }^{7}$ Center for Humanizing Critical Care, Intermountain Medical Center, Murray, Utah, USA

${ }^{8}$ Department of Internal Medicine, University of Utah School of Medicine, Salt Lake City, Utah, USA

Acknowledgements The authors thank the Beth Israel Deaconess Medical Center Patient and Family Advisory Council, whose members' insight improved this work, as well as the individuals who responded to this survey.

Contributors SKB, SMB: study conception; SDR: data acquisition; SKB, SDR, ALM, SMB: data analysis; SKB, SDR: manuscript writing. All authors had access to the data and critically revised the manuscript. SKB and SDR affirm that this manuscript is an honest, accurate and transparent account of the study being reported; that no important aspects of the study have been omitted; and that any discrepancies from the study as planned have been explained.

Funding This study was funded by Gordon and Betty Moore Foundation (10.13039/100000936).

Disclaimer The content is solely the responsibility of the authors and does not represent the official views of the funding agency, which had no role in the design and conduct of the study; the collection, management, analysis and interpretation of the data; or the preparation, review or approval of the manuscript.

Competing interests None declared.

Patient consent Not required. 
Ethics approval The Institutional Review Board (IRB) of Beth Israel Deaconess Medical Center approved this study.

Provenance and peer review Not commissioned; externally peer reviewed.

Data sharing statement No additional data available.

Open access This is an open access article distributed in accordance with the Creative Commons Attribution Non Commercial (CC BY-NC 4.0) license, which permits others to distribute, remix, adapt, build upon this work noncommercially, and license their derivative works on different terms, provided the original work is properly cited and the use is non-commercial. See: http://creativecommons.org/licenses/ by-nc/4.0/

(C) Article author(s) (or their employer(s) unless otherwise stated in the text of the article) 2018. All rights reserved. No commercial use is permitted unless otherwise expressly granted.

\section{REFERENCES}

1 The National Patient Safety Foundation's Lucian Leape Institute. Safety is personal: partnering with patients and families for the safest care. Boston: National patient safety foundation. 2014 http://c.ymcdn.com/sites/www.npsf.org/ resource/resmgr/LLI/Safety_Is_Personal.pdf (accessed 15 Mar 2017).

2 National Academy of Sciences, Engineering, and Medicine. Diagnostic team members and tasks: improving patient engagement and health care professional education and training in diagnosis. In: Balogh EP, Miller BT, Ball JR, eds. Improving diagnosis in health care. Washington, DC: National Academy of Sciences, 2015:145-216. (accessed 20 Jan 2017).

3 The National Patient Safety Foundation. RCA2: improving root cause analyses and actions to prevent harm (Version 2). 2016 http://www.npsf.org/?page=rca2 (accessed 20 Jan 2017).

4 Mazor KM, Smith KM, Fisher KA, et al. Speak up! addressing the paradox plaguing patient-centered care. Ann Intern Med 2016;164:618-9.

5 The National Patient Safety Foundation. National agenda for action: patients and families in patient safety nothing about me, without me. 2003 http://c.ymcdn.com/sites/www. npsf.org/resource/collection/ABAB3CA8-4E0A-41C5-A4806DE8B793536C/Nothing_About_Me.pdf (accessed 10 Jun 2017).

6 Etchegaray JM, Ottosen MJ, Burress L, et al. Structuring patient and family involvement in medical error event disclosure and analysis. Health Aff 2014;33:46-52.

7 Weissman JS, Schneider EC, Weingart SN, et al. Comparing patient-reported hospital adverse events with medical record review: do patients know something that hospitals do not? Ann Intern Med 2008;149:100-8.

8 Khan A, Furtak SL, Melvin P, et al. Parent-reported errors and adverse events in hospitalized children. JAMA Pediatr 2016;170:e154608.

9 Fisher K, Smith K, Gallagher T, et al. We want to know: eliciting hospitalized patients' perspectives on breakdowns in care. J Hosp Med 2017;12:603-9.

10 Fisher KA, Ahmad S, Jackson M, et al. Surrogate decision makers' perspectives on preventable breakdowns in care among critically ill patients: A qualitative study. Patient Educ Couns 2016;99:1685-93.

11 Davidson JE, Aslakson RA, Long AC, et al. Guidelines for family-centered care in the neonatal, pediatric, and adult ICU. Crit Care Med 2017;45:103-28.
12 Halpern NA, Pastores SM. Critical care medicine in the United States 2000-2005: an analysis of bed numbers, occupancy rates, payer mix, and costs. Crit Care Med 2010;38:65-71.

13 Frosch DL, May SG, Rendle KAS, et al. Authoritarian physicians and patients' fear of being labeled 'difficult' among key obstacles to shared decision making. Health Aff 2012;31:1030-8.

14 Delbanco T, Bell SK. Guilty, afraid, and alone--struggling with medical error. N Engl J Med 2007;357:1682-3.

15 Rainey H, Ehrich K, Mackintosh N, et al. The role of patients and their relatives in 'speaking up' about their own safety - a qualitative study of acute illness. Health Expectations $2015 ; 18: 392-405$.

16 Guinane J, Hutchinson AM, Bucknall TK. Patient perceptions of deterioration and patient and family activated escalation systems-A qualitative study. J Clin Nurs 2018;27:1621-31.

17 Kodali S, Stametz RA, Bengier AC, et al. Family experience with intensive care unit care: association of self-reported family conferences and family satisfaction. J Crit Care 2014;29:641-4.

18 Darer JD, Clarke DN, Sees AC, et al. Assessing the quality of death and dying in an integrated health care system in rural Pennsylvania. J Pain Symptom Manage 2015;50:343-9.

19 Johnson D, Wilson M, Cavanaugh B, et al. Measuring the ability to meet family needs in an intensive care unit. Crit Care Med 1998;26:266-71.

20 Netzer G, Sullivan DR. Recognizing, naming, and measuring a family intensive care unit syndrome. Ann Am Thorac Soc 2014;11:435-41.

21 Elliott D, Davidson JE, Harvey MA, et al. Exploring the scope of post-intensive care syndrome therapy and care: engagement of non-critical care providers and survivors in a second stakeholders meeting. Crit Care Med 2014;42:2518-26.

22 Bienefeld N, Grote N. Silence that may kill: When aircrew members don't speak up and why. Aviation Psychology and Applied Human Factors 2012;2:1-10.

23 Hamman WR. The complexity of team training: what we have learned from aviation and its applications to medicine. Qual Saf Health Care 2004;13(suppl 1):i72-i79.

24 Martinez W, Lehmann LS, Thomas EJ, et al. Speaking up about traditional and professionalism-related patient safety threats: a national survey of interns and residents. BMJ Qual Saf 2017;26:869-80.

25 Richard A, Pfeiffer Y, Schwappach DDL. Development and psychometric evaluation of the speaking up about patient safety questionnaire. J Patient Saf 2017:1.

26 Agency for Healthcare Research and Quality. TeamSTEPPS. https://www.ahrq.gov/teamstepps/index.html (accessed 20 Jan 2017).

27 Kolbe M, Burtscher MJ, Wacker J, et al. Speaking up is related to better team performance in simulated anesthesia inductions: an observational study. Anesth Analg 2012;115:1099-108.

28 Brady PW, Zix J, Brilli R, et al. Developing and evaluating the success of a family activated medical emergency team: a quality improvement report. BMJ Qual Saf 2015;24:203-11.

29 Eden EL, Rack LL, Chen LW, et al. Condition help: a patientand family-initiated rapid response system. J Hosp Med 2017;12:157-61.

30 Albutt AK, O'Hara JK, Conner MT, et al. Is there a role for patients and their relatives in escalating clinical deterioration in hospital? A systematic review. Health Expect 2017;20:818-25.

31 Gillespie A, Reader TW. The healthcare complaints analysis tool: development and reliability testing of a method for 
service monitoring and organisational learning. BMJ Qual Saf 2016;25:937-46.

32 de Vos MS, Hamming JF, Marang-van de Mheen PJ. The problem with using patient complaints for improvement. BMJ Qual Saf 2018;27:758-62.

33 Southwick FS, Cranley NM, Hallisy JA. A patient-initiated voluntary online survey of adverse medical events: the perspective of 696 injured patients and families. BMJ Qual Saf 2015;24:620-9.

34 Dana Farber Cancer Institute. Creating a culture of quality and safety. http://www.dana-farber.org/for-patients-and-families/ why-dana-farber/quality-and-patient-safety/creating-a-cultureof-quality-and-safety/ (accessed 15 Apr 2018).

35 Ginsburg L. 'Speaking up' climate: a new domain of culture to measure and explore. BMJ Qual Saf 2015;24:661-3.

36 Martinez W, Etchegaray JM, Thomas EJ, et al. 'Speaking up' about patient safety concerns and unprofessional behaviour among residents: validation of two scales. BMJ Qual Saf 2015;24:671-80.

37 The Joint Commission. Speak up initiatives. https://www. jointcommission.org/speakup.aspx (accessed 1 Jun 2017).

38 Medstar Health Institute for Quality and Safety. We Want to Know. https://www.medstariqs.org/research-programs/wewant-to-know/ (accessed 14 Feb 2018).

39 Agency for Health Care Research. Speak up campaign. https:// innovations.ahrq.gov/qualitytools/speak-campaign (accessed 15 Apr 2018).

40 Dyne LV, Ang S, Botero IC. Conceptualizing employee silence and employee voice as multidimensional constructs*. J Manag Stud 2003;40:1359-92.

41 Manapragada A, Bruk-Lee V. Staying silent about safety issues: conceptualizing and measuring safety silence motives. Accid Anal Prev 2016;91:144-56.

42 Morrison EW, Milliken FJ. Speaking up, remaining silent: the dynamics of voice and silence in organizations. Journal of Management Studies 2003;40:1353-8.

43 Okuyama A, Wagner C, Bijnen B. Speaking up for patient safety by hospital-based health care professionals: a literature review. BMC Health Serv Res 2014;14:1-8.

44 Lyndon A, Sexton JB, Simpson KR, et al. Predictors of likelihood of speaking up about safety concerns in labour and delivery. BMJ Qual Saf 2012;21:791-9.

45 Landgren R, Alawadi Z, Douma C, et al. Barriers of pediatric residents to speaking up about patient safety. Hosp Pediatr 2016;6:738-43.

46 Kobayashi H, Pian-Smith M, Sato M, et al. A cross-cultural survey of residents' perceived barriers in questioning/ challenging authority. Qual Saf Health Care 2006;15:277-83.

47 Schwappach DL, Gehring K. Frequency of and predictors for withholding patient safety concerns among oncology staff: a survey study. Eur J Cancer Care 2015;24:395-403.

48 Morrow KJ, Gustavson AM, Jones J. Speaking up behaviours (safety voices) of healthcare workers: A metasynthesis of qualitative research studies. Int J Nurs Stud 2016;64:42-51.

49 Barzallo Salazar MJ, Minkoff H, Bayya J, et al. Influence of surgeon behavior on trainee willingness to speak up: a randomized controlled trial. J Am Coll Surg 2014;219:1001-7.

50 Huang MY, Huston SA, Perri M. Consumer preferences for the predictive genetic test for Alzheimer disease. J Genet Couns 2014;23:172-8.

51 Brown SM, Bell SK, Roche SD, et al. Preferences of current and potential patients and family members regarding implementation of electronic communication portals in intensive care units. Ann Am Thorac Soc 2016;13:391-400.

52 Entwistle VA, McCaughan D, Watt IS, et al. Speaking up about safety concerns: multi-setting qualitative study of patients' views and experiences. Qual Saf Health Care 2010;19:e33.

53 Beal DJ. SAS code to select the best multiple linear regression model for multivariate data using information criteria. SAS Glob Forum 2005. http://analytics.ncsu.edu/sesug/2005/SA01_ 05.PDF (accessed 30 May 2017).

54 EuroQol Group. EuroQol--a new facility for the measurement of health-related quality of life. Health Policy 1990;16:199-208.

55 Schwappach DL, Wernli M. Am I (un)safe here? Chemotherapy patients' perspectives towards engaging in their safety. Qual Saf Health Care 2010;19:e9.

56 Bosslet GT, Pope TM, Rubenfeld GD, et al. An Official ATS/ AACN/ACCP/ESICM/SCCM Policy Statement: Responding to Requests for Potentially Inappropriate Treatments in Intensive Care Units. Am J Respir Crit Care Med 2015;191:1318-30.

57 Davis RE, Jacklin R, Sevdalis N, et al. Patient involvement in patient safety: what factors influence patient participation and engagement? Health Expect 2007;10:259-67.

58 The National Patient Safety Foundation's Lucian Leape Institute. Shining a light: safer health care through transparency. 2015 http://c.ymcdn.com/sites/www.npsf. org/resource/resmgr/LLI/Shining-a-Light_Transparency.pdf (accessed 4 Jun 2017).

59 Redley B, McTier L, Botti M, et al. Patient participation in inpatient ward rounds on acute inpatient medical wards: a descriptive study. BMJ Qual Saf 2018:bmjqs-2017-007292 (Epub ahead of print 23 Feb 2018).

60 Wyskiel RM, Weeks K, Marsteller JA. Inviting families to participate in care: a family involvement menu. Jt Comm J Qual Patient Saf 2015;41:43-6.

61 American Medical Association. Listening with empathy [continuing medical education module]. https://www. stepsforward.org/modules/empathetic-listening (accessed 15 Feb 2018).

62 Schwappach DL, Gehring K. Silence that can be dangerous: a vignette study to assess healthcare professionals' likelihood of speaking up about safety concerns. PLoS One 2014;9:e104720-8.

63 Morrison EW. Employee voice behavior: integration and directions for future research. Acad Manag Ann 2011;5:373-412.

64 Vincent CA, Coulter A. Patient safety: what about the patient? Qual Saf Health Care 2002;11:76-80.

65 Delbanco T, Berwick DM, Boufford JI, et al. Healthcare in a land called peoplepower: nothing about me without me. Health Expect 2001;4:144-50.

66 World Health Organization. Patients for patient safety. http:// www.who.int/patientsafety/patients_for_patient/en/ (accessed 1 Jun 2017).

67 Davis RE, Koutantji M, Vincent CA. How willing are patients to question healthcare staff on issues related to the quality and safety of their healthcare? An exploratory study. Qual Saf Health Care 2008;17:90-6.

68 Mazor KM, Roblin DW, Greene SM, et al. Toward patientcentered cancer care: patient perceptions of problematic events, impact, and response. J Clin Oncol 2012;30:1784-90. 